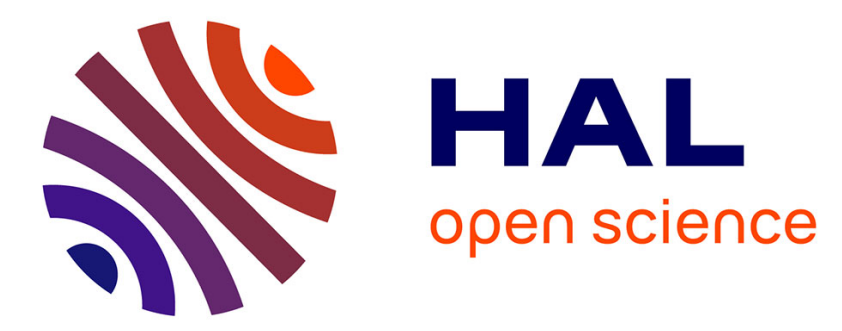

\title{
Stochastic analysis of energy savings with sleep mode in OFDMA wireless networks
}

\author{
Dimitrios Tsilimantos, Jean-Marie Gorce, Eitan Altman
}

\section{To cite this version:}

Dimitrios Tsilimantos, Jean-Marie Gorce, Eitan Altman. Stochastic analysis of energy savings with sleep mode in OFDMA wireless networks. IEEE INFOCOM - 32nd International Conference on Computer Communications, Apr 2013, Turin, Italy. pp.1097-1105, 10.1109/INFCOM.2013.6566900 . hal-00913219

\section{HAL Id: hal-00913219 \\ https://hal.inria.fr/hal-00913219}

Submitted on 8 Dec 2013

HAL is a multi-disciplinary open access archive for the deposit and dissemination of scientific research documents, whether they are published or not. The documents may come from teaching and research institutions in France or abroad, or from public or private research centers.
L'archive ouverte pluridisciplinaire HAL, est destinée au dépôt et à la diffusion de documents scientifiques de niveau recherche, publiés ou non, émanant des établissements d'enseignement et de recherche français ou étrangers, des laboratoires publics ou privés. 


\title{
Stochastic Analysis of Energy Savings with Sleep Mode in OFDMA Wireless Networks
}

\author{
Dimitrios Tsilimantos*, Jean-Marie Gorce* and Eitan Altman ${ }^{\dagger}$ \\ *INRIA, University of Lyon \\ INSA-Lyon, CITI-INRIA, F-69621, Villeurbanne, France \\ †INRIA, Sophia Antipolis \\ 10 route des Lucioles, 06902, Sophia Antipolis, France \\ Email: \{dimitrios.tsilimantos, jean-marie.gorce\}@insa-lyon.fr, eitan.altman@inria.fr
}

\begin{abstract}
The issue of energy efficiency (EE) in Orthogonal Frequency-Division Multiple Access (OFDMA) wireless networks is discussed in this paper. Our interest is focused on the promising concept of base station (BS) sleep mode, introduced recently as a key feature in order to dramatically reduce network energy consumption. The proposed technical approach fully exploits the properties of stochastic geometry, where the number of active cells is reduced in a way that the outage probability, or equivalently the signal to interference plus noise (SINR) distribution, remains the same. The optimal EE gains are then specified with the help of a simplified but yet realistic BS power consumption model. Furthermore, the authors extend their initial work by studying a non-singular path loss model in order to verify the validity of the analysis and finally, the impact on the achieved user capacity is investigated. In this context, the significant contribution of this paper is the evaluation of the theoretically optimal energy savings of sleep mode, with respect to the decisive role that the BS power profile plays.
\end{abstract}

\section{INTRODUCTION}

As the demand for increased performance of wireless networks has risen beyond all expectations over the last years, the need for rapid evolution of communication systems became apparent. Now that the third generation (3G) networks has been deployed throughout the world, the main interest of the wireless communications industry and scientific community migrates towards fourth generation systems (4G). However, the explosion of data traffic with billions of daily mobile users, along with the required exponential growing scale of network infrastructure, lead to the continuous increase of energy consumption. In this direction, most of the network operators are gradually showing more interest in order to limit their electricity bill that represents a large portion of their operational expenditures (OPEX). Furthermore, from an equally important environmental perspective, according to the International Communication Union (ITU), the ICT industry is currently responsible for almost the $2.5 \%$ of global greenhouse gas emissions and this contribution is expected to double in near future [1]. Sustainable development has become an undeniable requirement nowadays and communication systems should aim to improve their green footprint, a paradigm shift that would directly benefit other energy-intensive industry sectors as well.

The truth is that EE has not attracted the necessary attention as an optimization objective for wireless networks so far, since today's systems are mainly designed for optimal capacity. As a consequence, green radio has emerged as a widely recognized and essential research direction towards a holistic approach for energy efficient networks and several significant actions are already committed to this goal. For instance, the Next Generation Mobile Networks (NGMN) Alliance and its Green Telco Initiative [2] bring together partners with green activities and the EARTH FP7 project investigates the development of a new energy efficient wireless generation [3]. More recently, the mission of the GreenTouch Initiative is to deliver the architecture, specifications and roadmap in order to increase total network EE by a factor of 1000 from current levels [4].

It is well known that most of the energy is consumed by the BSs in cellular networks. Moreover, in low traffic scenarios, for example during the night, the situation is substantially worse in terms of EE, since BSs still consume a significant amount of energy, even if their transmit power is really low and they may not actually serve any user. In order to overcome these challenges and satisfy the urgent demands for $\mathrm{EE}$, the promising concept of BS sleep mode is introduced recently. Significant savings can be realized from monitoring the loading variations over time, allowing the network resources to dynamically match the traffic demand as close as possible by entering a low-power sleep mode. This idea is motivated by the fact that there is an important difference in consumed power between BS active and sleep mode. It should be clear at this point that this technique brings new challenges that must be addressed without compromising other performance indicators, in particular related to coverage issues.

Several approaches can be found in the open literature considering different aspects of this research topic. An interesting investigation of sleep mode techniques that improve cellular radio EE is performed in [5], [6]. The same problem is considered in [7], where the authors focus on sleep mode for small cell deployments. The switch-off transients in one cell are studied in [8], while the authors in [9] consider the impact of energy saving on the uplink transmit power. Apart from the performance within one single network, an additional challenge can also be introduced when an entire system with multiple operators is examined. For example, energy-aware system selection schemes for cooperative $2 \mathrm{G} / 3 \mathrm{G}$ networks and for two operators offering service over the same area are presented in [10] and [11] respectively. Finally, other representative efforts 
to enhance EE based on techniques besides the BS sleep mode can be found in [12]-[14].

The scope of this paper is to take a step forward and study the impact of the BS power model profile on the optimal achieved EE gains when the sleep mode feature is applied in an OFDMA network. The proposed analysis provides a way to calculate the overall network energy savings by reducing the number of active BSs in a way that the outage probability remains the same. Specifically, the authors build upon a rather simplified but yet realistic model for power consumption, that maps the radiated power to the total power supply of a BS at variable loads and study the case where the random location of BSs forms a Poisson point process (PPP). The main reason for the adoption of the PPP model is that its independence property, combined with the random spatial distribution of the BS positions, allows the utilization of strong tools from stochastic geometry in order to characterize key metrics, such as the outage probability and the average user capacity [15]-[18]. Moreover, special concern is given to constrain the possibly increased transmit power of active BSs below a specified threshold, an aspect that can also be aligned to the growing concerns among the authorities and the general public for potential adverse health effects of radiofrequency (RF) radiation [19]. Then, the initial study framework is expanded to the case of a non-singular path loss model in order to verify the validity of our theoretical model, motivated by the fact that the most commonly used power law path loss model can lead to inaccurate results under certain conditions. Finally, since this methodology is focused on coverage, the impact on the user capacity is also investigated. In any case, the evaluation of the optimal number of active BSs and the respective energy savings is performed with extended parametric studies, where various scenarios can easily be examined.

The remainder of the paper is organized as follows: Section II describes the BS power consumption model which is undoubtedly necessary for any EE evaluation. In Section III, the mathematical model, including the analysis of the outage probability, the capacity, the sleep mode and the application of a modified non-singular path loss model is discussed. Then, the theoretically optimal network energy savings are evaluated in Section IV. The numerical results are presented in Section V and finally, concluding remarks are made in Section VI.

\section{Base Station Power Consumption Model}

In order to verify and assess the potential EE improvements, a suitable BS power consumption model should be defined. Since the actual power consumption breakdown can be too complicated, a rather simplified model needs to be introduced, able to capture the main dependencies, such as the key relation between the total consumed power and the output power transmitted to serve system users. Several examples of models that have recently been proposed in this line of thought can be found in [10], [20]-[22]. Generally, the components that contribute to overall BS power consumption can be separated into two fundamental parts. The first one describes the power figure that is independent of the radiated power, primarily as

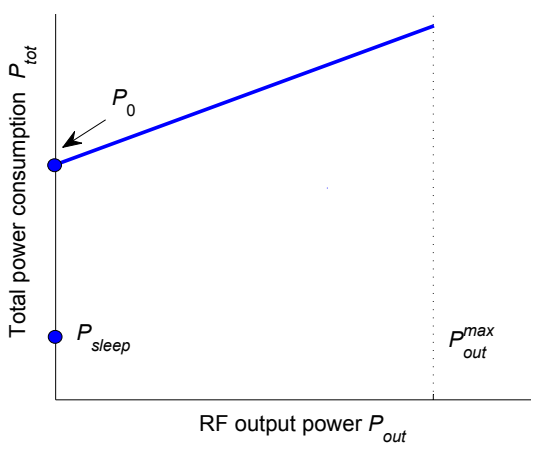

Fig. 1. Linear BS power consumption model.

a result of the signal processing, the battery backup supply and the contribution from other circuit elements, such as the analog-to-digital converters and the filters. On the other hand, the second part represents the portion that scales with the output power and it is clearly dominated by the presence of the energydemanding power amplifier. The model should also take into account the substantial losses from the main power supply and the site cooling system, which are considered to scale linearly with the consumption of the above parts. Hence, even though additional parameters in more detail could easily be included, the total BS power consumption $P_{t o t}$ can be expressed as:

$$
P_{\text {tot }}=\frac{N_{t}\left(\frac{P_{\text {out }}}{\eta_{\text {pa }}}+P_{s p}\right)+P_{\text {site }}}{\left(1-C_{\text {supply }}\right)\left(1-C_{\text {cooling }}\right)}
$$

where $N_{t}$ is the number of antennas, $P_{\text {out }}$ is the transmit power at variable load, $\eta_{p a}$ is the efficiency of the power amplifier, $P_{s p}$ and $P_{\text {site }}$ are the power consumption of all the other parts of the RF chain and the BS site respectively, and finally $C_{\text {supply }}$ and $C_{\text {cooling }}$ denote the losses from the main supply and the cooling device accordingly. One should notice that this model is suitable for various BS types by simply applying the right parameter values. The adopted power consumption model is defined by further simplifying the previous equation in a similar way with [20], according to the linear approximation:

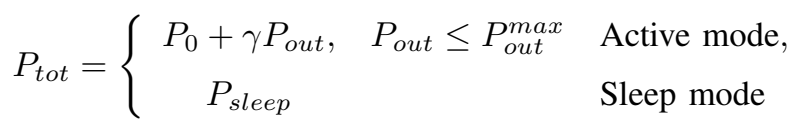

where $P_{0}$ is the minimum consumption of an active BS, $\gamma$ is the slope of the traffic-dependent part, $P_{\text {out }}^{\max }$ is the maximum output power constraint and $P_{\text {sleep }}$ is the power consumption in sleep mode. Fig. 1 illustrates the above model that is used for formulation purposes throughout the remainder of the paper. This figure also implies the motivation behind the potential EE gains introduced by sleep mode, i.e. $P_{\text {sleep }}$ is significantly lower than $P_{0}$, which is actually the case for all BS types.

\section{Mathematical Model}

A large-scale OFDMA cellular network is considered in this section, where specifically the random distribution of BSs and users form two independent 2D homogeneous PPPs $\Phi_{b}$ and $\Phi_{u}$ with intensities $\lambda_{b}$ and $\lambda_{u}$ respectively. This approach is very popular among other stochastic models due to its well known properties and leads to a topology of various cell shapes and 
sizes that usually approximates the real deployment in large wireless networks. Our interest is focused on the downlink direction with full frequency reuse, where the users are served by their nearest BS and hence, the number of cell users is equal to $M=\lambda_{u} / \lambda_{b}$ [17]. All BSs are assumed to have the same total available bandwidth $W$ equally shared among the $M$ users. Thereby, a scenario with full resource utilization is obtained, where each mobile is allocated $w=W / M$ bandwidth and receives interference from all the points of $\Phi_{b}$ besides the one it is connected, i.e. the closest one. Firstly, the theoretical model for the outage probability and the capacity is given following a similar analysis with [16]-[18] and then our corollaries for the sleep mode and the non-singular path loss model are presented.

\section{A. Outage Probability}

A key performance indicator for wireless systems is the SINR level measured at the receiver, which is defined by:

$$
S I N R=\frac{S}{N_{u}+I}, \quad \text { with } N_{u}=w N_{0}
$$

where $S$ is the desired dedicated signal, $N_{u}$ is the noise power for bandwidth $w, N_{0}$ is the noise power spectral density and $I$ is the interference. Note that both $S$ and $I$ signals are subject to the stochastic nature of the PPP. Moreover, the signal $S$ can be described in more detail according to:

$$
S=P_{t} h_{S} \ell(R)
$$

where $P_{t}$ is the link transmit power, $h_{S}$ is a random variable that incorporates the effect of fading and $\ell(R)$ is the path loss function, assumed to depend only on the distance $R$ between a mobile user and the serving BS. The coverage probability $P_{C}$ associated to a specific SINR threshold $\rho$ is then given by:

$$
P_{C} \triangleq \mathbb{P}[\operatorname{SINR}>\rho]=\mathbb{P}\left[h_{S}>\frac{\rho\left(N_{u}+I\right)}{P_{t} \ell(R)}\right]
$$

and the outage probability or equivalently the SINR cumulative distribution function (cdf) is equal to $1-P_{C}$. In the typical case of Rayleigh fading, the variable $h_{S}$ follows an exponential distribution and therefore, for a mean value $\mathbb{E}\left[h_{S}\right]=1 / \tau$ :

$$
P_{C}=e^{-s N_{u}} \mathbb{E}\left[e^{-s I}\right], \text { with } s \triangleq \frac{\tau \rho}{P_{t} \ell(R)}
$$

At this point (6) introduces the Laplace transform of interference $\mathcal{L}_{I}(s) \triangleq \mathbb{E}\left[e^{-s I}\right]$, which is given by:

$$
\mathcal{L}_{I}(s)=\mathbb{E}\left[\exp \left(-s \sum_{i \in \Phi_{b} \backslash b_{u}} P_{t} h_{I, i} \ell\left(r_{i}\right)\right)\right]
$$

where $h_{I}$ represents the fading coefficient of the interferers and the summation is performed over any interfering BS $i$, i.e. all the points of $\Phi_{b}$ except the serving BS $b_{u}$, with respective distance $r_{i}$ from the mobile user. Finally, the expectation is taken over both the fading and the point process, and since these random distributions are independent, (7) becomes:

$$
\begin{aligned}
& \mathcal{L}_{I}(s)=\mathbb{E}_{\Phi}\left[\prod_{i \in \Phi_{b} \backslash b_{u}} \mathbb{E}_{h}\left[e^{-s P_{t} h_{I, i} \ell\left(r_{i}\right)}\right]\right] \\
& =\exp \left\{-\int_{R}^{\infty}\left(1-\mathbb{E}_{h}\left[e^{-s P_{t} h_{I} \ell(r)}\right]\right) 2 \pi \lambda_{b} r \mathrm{~d} r\right\}
\end{aligned}
$$

where the last step is derived through the fact that $\mathcal{L}_{I}$ is expressed as a probability generating functional (PGFL), [15]. Notice that the integration limits are from $R$ to $\infty$, as the mobile is connected to the nearest BS $b_{u}$ and all the interfering BSs cannot be any closer. By assuming Rayleigh fading with mean value $1 / \tau$ for the interfering signals as well, the Laplace transform $\mathcal{L}_{h}(s)=\frac{\tau}{\tau+s}$ of the random variable $h_{I}$ yields:

$$
\mathcal{L}_{I}(s)=\exp \left(-2 \pi \lambda_{b} \int_{R}^{\infty} \frac{r}{1+\frac{\tau}{s P_{t} \ell(r)}} \mathrm{d} r\right)
$$

It should be mentioned that even in the more complex case where the channel of the interferers is subject to any type of fading [18], the respective theoretical analysis is similar and our conclusions can easily be proved to be valid. In order to further analyze the stochastic interference term, the path loss model needs to be defined. In this direction, the following simple power law function is considered initially:

$$
P L 1: \ell(r)=(k r)^{-a}
$$

where $k>0$ is a scenario dependent constant and $a>2$ is the path loss exponent. Despite its simplicity, this model is quite accurate for large distances and it facilitates significantly the remainder of the analysis. The next step is taken by replacing the argument $s$ of $\mathcal{L}_{I}$ according to (6), that leads to the result:

$$
\begin{aligned}
& P_{C}\left(\lambda_{b} \mid R\right)=\exp \left\{-\frac{\tau \rho N_{u}}{P_{t}}(k R)^{a}-\pi \lambda_{b} R^{2} I(a, \rho)\right\} \\
& I(a, \rho) \triangleq \int_{1}^{\infty} \frac{\rho}{\rho+z^{a / 2}} \mathrm{~d} z=\frac{2 \rho}{a-2}{ }_{2} F_{1}\left(1,1-\frac{2}{a} ; 2-\frac{2}{a} ;-\rho\right)
\end{aligned}
$$

after the substitution $(r / R)^{2} \rightarrow z$, where ${ }_{2} F_{1}$ is the Gaussian hyper-geometric function.

It should be clear that the coverage probability is defined so far as a function of distance $R$. However, there are some well known properties that characterize this variable in the case of a PPP that can be easily exploited. For a randomly located user, the cdf of the distance $R$ to the closest point of a PPP is:

$$
\mathbb{P}[R \leq r]=1-e^{-\pi \lambda_{b} r^{2}}, \text { with } \mathbb{E}[R]=\frac{1}{2 \sqrt{\lambda_{b}}}
$$

As a result, the final expression of the average area coverage 
probability is given by:

$$
\begin{aligned}
& P_{C}\left(\lambda_{b}\right)=2 \pi \lambda_{b} \int_{0}^{\infty} P_{C}\left(\lambda_{b} \mid R\right) e^{-\pi \lambda_{b} R^{2}} R \mathrm{~d} R \stackrel{(11)}{=} \\
& \pi \int_{0}^{\infty} \exp \left\{-\frac{\tau \rho N k^{a}}{P_{t}} \frac{\lambda_{b}}{\lambda_{u}}\left(\frac{x}{\lambda_{b}}\right)^{\frac{a}{2}}-\pi x[1+I(a, \rho)]\right\} \mathrm{d} x
\end{aligned}
$$

where $N=W N_{0}$ and $N_{u}$ is replaced by $N / M$. The last part is derived with the substitution $\lambda_{b} R^{2} \rightarrow x$ that limits the impact of parameter $\lambda_{b}$ to the first part of the integral (SNR term). Notice that the second part (SIR term) does not depend on $\lambda_{b}$, due to the fact that the variation of the required signal power for different cell sizes is counter-balanced by the respective one of the received interference.

\section{B. Capacity}

Besides the performance evaluation in terms of outage probability, the achieved average user capacity $C_{u}$ consists another critical objective that should be included in our approach. By using Shannon capacity formula with interference treated as noise, $C_{u}$ is given by:

$$
C_{u}=2 \pi \lambda_{b} \int_{0}^{\infty} \mathbb{E}\left[w \log _{2}\left(1+\frac{1}{\Gamma} \operatorname{SINR}\right)\right] e^{-\pi \lambda_{b} R^{2}} R \mathrm{~d} R
$$

where $\Gamma$ is the SINR gap that introduces the impact of practical modulation and coding schemes. Since the argument of the expectation in (14) is a positive random variable $\mathrm{X}$, by using the property $\mathbb{E}[X]=\int_{0}^{\infty} \mathbb{P}[X>\xi] d \xi$ the analysis is similar to the one for outage probability, leading to:

$$
\begin{gathered}
C_{u}=\pi \int_{0}^{\infty} \int_{0}^{\infty} \exp \left\{-\frac{\tau N k^{a} \theta}{P_{t}} \frac{\lambda_{b}}{\lambda_{u}}\left(\frac{x}{\lambda_{b}}\right)^{\frac{a}{2}}\right\} e^{-\pi x[1+I(a, \theta)]} \mathrm{d} \xi \mathrm{d} x \\
\text { with } \theta=\Gamma\left[\exp \left(\frac{\xi \ln 2}{W} \frac{\lambda_{u}}{\lambda_{b}}\right)-1\right]
\end{gathered}
$$

This expression may require the computation of multiple numerical integrations, but it allows us to study the impact of sleep mode on achieved capacity for various set of parameters.

\section{Sleep Mode}

Our key corollary regarding the stochastic theoretical model is presented here. As mentioned before, an operator should aim to increase network energy savings by keeping some BSs in sleep mode. A possible implementation of this concept is to select and keep a point of the stochastic process with activation probability $0 \leq \beta \leq 1$. Therefore, a fraction $\beta$ of all the BSs is kept in active mode, while the remaining $1-\beta$ are switchedoff. This procedure is known as the PPP thinning property that leads to a new PPP with intensity $\beta \lambda_{b}$. In our model, this also results into an increased number of cell users $M / \beta$ with reduced bandwidth $\beta w$ and the coverage probability becomes:

$$
\begin{aligned}
& P_{C}\left(\beta \lambda_{b}\right)= \\
& \pi \int_{0}^{\infty} \exp \left\{-\beta^{1-\frac{a}{2}} \frac{\tau \rho N k^{a}}{P_{t}} \frac{\lambda_{b}}{\lambda_{u}}\left(\frac{x}{\lambda_{b}}\right)^{\frac{a}{2}}\right\} e^{-\pi x[1+I(a, \rho)]} \mathrm{d} x(16)
\end{aligned}
$$

Combining (13),(16), one can observe that if $P_{t}$ increases by $\beta^{1-\frac{a}{2}}$, the outage probability remains the same. This is achieved at the cost of higher total BS transmit power, increased from $P_{\text {out }}=M P_{t}$ to $P_{\text {out }}^{\prime}=(M / \beta) P_{t} \beta^{1-\frac{a}{2}}=P_{\text {out }} / \beta^{\frac{a}{2}}$, as described in the following corollary:

Corollary 1: Sleep mode scaling property

$$
\left.P_{C}\left(\lambda_{b}\right)\right|_{P_{\text {out }}}=\left.P_{C}\left(\beta \lambda_{b}\right)\right|_{P_{\text {out }} f(a, \beta)} \text {, if } f(a, \beta)=\beta^{-\frac{a}{2}}
$$

This remark provides a useful way to determine the optimal energy savings by reducing the number of active BSs without increasing the outage probability. However, keep in mind that the user capacity $C_{u}$ is lower for $\beta \lambda_{b}$ according to (14), even if SINR has the same level, due to the reduced user bandwidth. Therefore, the impact on the user capacity should not be neglected. Finally, it should be noted that the same corollary can be derived if instead of using the thinning property, a transformation of the space and specifically the dilation by the factor $\beta^{-\frac{1}{2}}$ is performed on the initial PPP. This approach is actually considered in [23], but an analytical expression for the SINR distribution is not provided.

\section{Modified Path loss Model}

Although the path loss model of (10) is commonly used in the literature, it is not appropriate for small distances due to its singularity for $r=0$. This pole can even lead to invalid scenarios where the received power exceeds the transmitted one, as a mobile moves closer to a BS. In order to avoid this inaccuracy, a modified model should be introduced that could allow us to verify the validity of the previous analysis, since the computation of the outage probability and the user capacity includes these small distances (see integration limits in (13),(15)). To this end, by assuming a BS antenna height $L$, a simple non-singular path loss model is obtained:

$$
P L 2: \ell(r)=(k d)^{-a}, \text { with } d=\sqrt{L^{2}+r^{2}}
$$

while several other possible choices of models can be found for example in [17], [24]. The new expression of probability $P_{C}$ is found in the same way as for $P L 1$, leading to:

$$
\begin{aligned}
& P_{C}\left(\lambda_{b}\right)=\pi e^{\pi \lambda_{b} L^{2}} . \\
& \int_{\lambda_{b} L^{2}}^{\infty} \exp \left\{-\frac{\tau \rho N k^{a}}{P_{t}} \frac{\lambda_{b}}{\lambda_{u}}\left(\frac{x}{\lambda_{b}}\right)^{\frac{a}{2}}-\pi x[1+I(a, \rho)]\right\} \mathrm{d} x
\end{aligned}
$$

and the average user capacity $C_{u}$ becomes:

$$
\begin{aligned}
& C_{u}=\pi e^{\pi \lambda_{b} L^{2}} . \\
& \int_{\lambda_{b} L^{2}}^{\infty} \int_{0}^{\infty} \exp \left\{-\frac{\tau N k^{a} \theta}{P_{t}} \frac{\lambda_{b}}{\lambda_{u}}\left(\frac{x}{\lambda_{b}}\right)^{\frac{a}{2}}\right\} e^{-\pi x[1+I(a, \theta)]} \mathrm{d} \xi \mathrm{d} x
\end{aligned}
$$

The above equations provide a way to evaluate the deviation from (13),(15) respectively for different parameter values. 
However, it is interesting to verify that for a network that is not too dense and $\lambda_{b}$ is small enough compared to $1 / L^{2}$, then the term $\lambda_{b} L^{2} \rightarrow 0$ and the singularity of $P L 1$ does not affect the analysis, as the next corollary summarizes:

Corollary 2: Convergence of path loss models

$$
\left.\left.\left[P_{C}\left(\lambda_{b}\right), C_{u}\right]\right|_{P L 2} \approx\left[P_{C}\left(\lambda_{b}\right), C_{u}\right]\right|_{P L 1}, \text { if } \quad \lambda_{b} \ll \frac{1}{L^{2}}
$$

This property is also confirmed from our results indicating that the difference by using the modified path loss model $P L 2$ is marginal for a large range of parameter values, facilitating the evaluation of the optimal EE gains.

\section{Optimal ENERgy SAVINGS}

Given the definition of the BS power consumption model from (2) and the theoretical analysis of the previous section, a theoretical framework that comprises the main contribution of this paper is provided, enabling the evaluation of the optimal number of active BSs in terms of $\mathrm{EE}$. In order to characterize the achieved EE gains, an energy consumption index must be employed. An appropriate metric that focuses on total network energy consumption and coverage issues is the area power density, expressed in $W / m^{2}$. Thus, the EE of the initial network with all the BSs in active mode is:

$$
E E_{\text {init }}=\lambda_{b}\left(P_{0}+\gamma P_{\text {out }}\right)
$$

In sleep mode, a fraction $\beta$ of the BSs is kept in active mode and $P_{\text {out }}$ is increased according to Corollary 1, leading to:

$$
E E_{\text {sleep }}=\lambda_{b} \beta\left[P_{0}+\gamma P_{\text {out }} f(a, \beta)\right]+\lambda_{b}(1-\beta) P_{\text {sleep }}
$$

while the achieved EE gains can then be defined by the ratio:

$$
G \triangleq \frac{E E_{\text {init }}}{E E_{\text {sleep }}}=\frac{P_{0}+\gamma P_{\text {out }}}{\beta\left[P_{0}+\gamma P_{\text {out }} f(a, \beta)\right]+(1-\beta) P_{\text {sleep }}}
$$

Notice that $G$ is independent from BS density $\lambda_{b}$, but one should keep in mind that $P_{t}$, and hence $P_{\text {out }}$, is actually specified according to $\lambda_{b}$ in order to achieve a specific outage probability. From (22), since $G$ is a function of probability $\beta$, the following optimization problem is formed:

Problem 1: Optimal number of active BSs

$$
\begin{aligned}
& \text { find } \beta_{\text {opt }}=\arg \max _{\beta}(G) \\
& \text { s.t. } 1 . \quad P_{\text {out }} f(a, \beta) \leq P_{\text {out }}^{\max } \\
& \text { 2. } \quad 0 \leq \beta \leq 1
\end{aligned}
$$

The first constraint sets the threshold value $\beta_{t h r}$ with respect to the maximum allowed transmit power and the second one obviously follows from the fact that $\beta_{\text {opt }}$ represents a

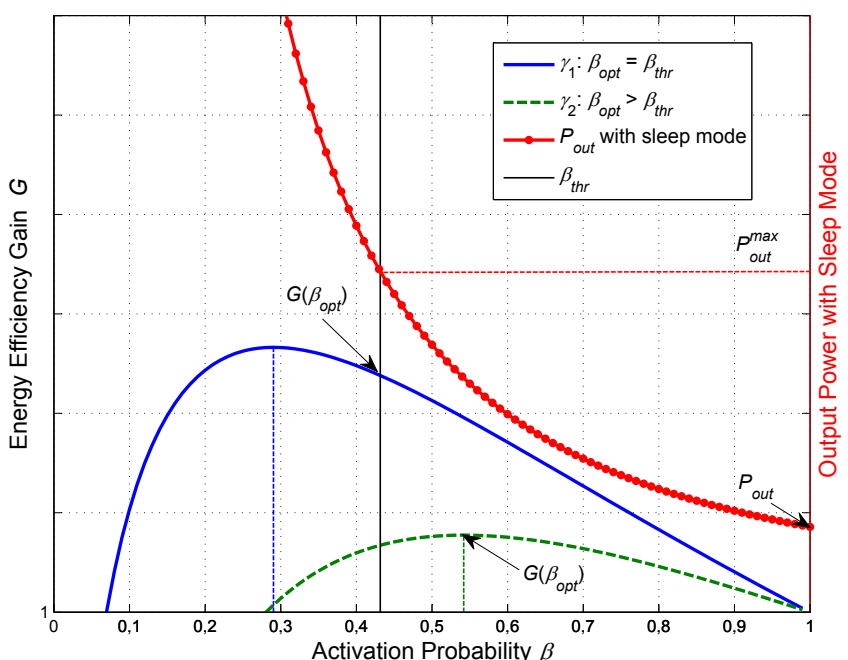

Fig. 2. Illustration of the optimal energy efficiency gains for 2 different set of parameters, including the applied max transmit power constraint.

probability value. The solution of this problem defines the optimal EE gains $G\left(\beta_{\text {opt }}\right)$ that are achieved for:

$$
\begin{gathered}
\beta_{\text {opt }}=\min \left(1, \max \left(\left[\frac{\gamma P_{\text {out }}}{P_{0}-P_{\text {sleep }}}\left(\frac{a}{2}-1\right)\right]^{\frac{2}{a}}, \beta_{\text {thr }}\right)\right) \\
\text { with } \beta_{\text {thr }} \triangleq\left(\frac{P_{\text {out }}}{P_{\text {out }}^{\text {max }}}\right)^{\frac{2}{a}}
\end{gathered}
$$

An example is shown in Fig.2, for two different values of parameter $\gamma$ with $\gamma_{1}<\gamma_{2}$, where the EE gains are plotted as a function of probability $\beta$. The right $\mathrm{y}$-axis is introduced to show the dependency of the output power that determines the admissible region. As this figure shows, in the case of the first curve for $\gamma_{1}$, the solution is $\beta_{o p t}=\beta_{t h r}$ since the maximum gain is achieved for a value below this threshold. On the other hand, the second curve admits the maximum gain for a value of $\beta$ above $\beta_{t h r}$ and thus, this is accepted as the optimal one. Finally, it should be highlighted that the non-monotonic shape of the curves indicates the existing trade-off between the energy savings from switching off BSs and the cost of the increased power transmitted by the remaining active ones.

\section{Numerical Results}

The numerical results for the proposed analytical framework are presented in this section. Since this evaluation depends on many variables, different cases are studied where some parameters vary, while the remaining ones, unless otherwise stated, are equal to their default representative values, summarized here in Table I. All the figures provide results for different values of exponent $a$ reflecting various propagation conditions and moreover, the respective constant $k$ is defined in such a way that for $a=3.5$, the obtained path losses are similar to the ones from the COST-Hata propagation prediction model for a medium sized city. Further information regarding the power model parameters can be found for example in [20], where the authors provide several values for a typical simulation scenario of a Long Term Evolution (LTE) system. 
TABLE I

Mathematical Model Parameters

\begin{tabular}{|l||l|}
\hline Total BS bandwidth & $W=20 \mathrm{MHz}$ \\
\hline Noise power spectral density & $N_{0}=-174 \frac{\mathrm{dBm}}{\mathrm{Hz}}$ \\
\hline PPP BS intensity & $\lambda_{b}=1$ point $/ \mathrm{km}^{2}$ \\
\hline PPP user intensity & $\lambda_{u}=40$ points $/ \mathrm{km}^{2}$ \\
\hline BS output link power & $P_{t}=20 \mathrm{dBm}$ \\
\hline Fading mean value & $1 / \tau=1$ \\
\hline SINR gap & $\Gamma=2$ \\
\hline Path loss exponent & $a \in[2.5,5]$ \\
\hline Path loss model constant & $k=8.4 \mathrm{~m}^{-1}$ \\
\hline BS antenna height & $L=30 \mathrm{~m}$ \\
\hline Max. total allowed BS output power & $P_{\text {out }}^{\text {max }}=40 \mathrm{~W}$ \\
\hline Min. consumption in active mode & $P_{0}=100 \mathrm{~W}$ \\
\hline Consumption in sleep mode & $P_{\text {sleep }}=1 \mathrm{~W}$ \\
\hline Power slope & $\gamma=4$ \\
\hline
\end{tabular}

Firstly, the performance in terms of outage probability is studied. In this line of thought, Fig. 3 presents the outage probability $P_{n C}=1-P_{C}$ as a function of the SINR threshold $\rho$. It is interesting to see that $P_{n C}$ is not a monotonic function of path loss exponent $a$. Indeed, starting from a value of $a=2.5$, $P_{n C}$ is initially reduced for $a=3.5$ and then increases for higher values. This behavior is based on the nature of (13), where it is easily found that as a admits lower values, the interference is increased and the SIR term becomes dominant. On the other hand, if $a$ is higher, then the level of the received signal drops quickly and thus the SNR term turns out to be responsible for the degradation of $P_{n C}$. Obviously, an optimal value of $a$ with respect to $P_{n C}$ lies between the two extremes. One should also observe that the estimated outage probability is quite high, indicating in particular a value of $55 \%$ for the typical case of $\rho=0 \mathrm{~dB}$ and $a=3.5$, which is obviously not acceptable from an operator's point of view. This is explained by the fact that the currently adopted theoretical model does not incorporate cell sectors with directional antennas or any other interference reduction technique, including for example frequency reuse approaches or gains from more sophisticated multiple antennas schemes. Nevertheless, besides the fact that these features could provide more realistic results, our analysis and conclusions are still valid.

Furthermore, the outage probability as a function of BS link output power $P_{t}$ is illustrated in Fig.4. Two different cases are examined for $\rho=[-10,0] \mathrm{dB}$. It is clear from this figure that $P_{n C}$ is decreased as $P_{t}$ admits higher values. However, this trend holds until a certain point, where the additional increase of $P_{t}$ has no positive effect. This is more evident as $a$ gradually decreases and the region where the SIR term becomes dominant is reached, since according to (13), $P_{t}$ has no impact on this term. In other words, it is not meaningful to increase the transmit power at interference-limited scenarios in order to reduce outage probability and other interference reduction techniques should be applied. It is also easy to verify that above a reasonable value of $P_{t}$, as previously, the best results are obtained for the intermediate value of $a=3.5$.

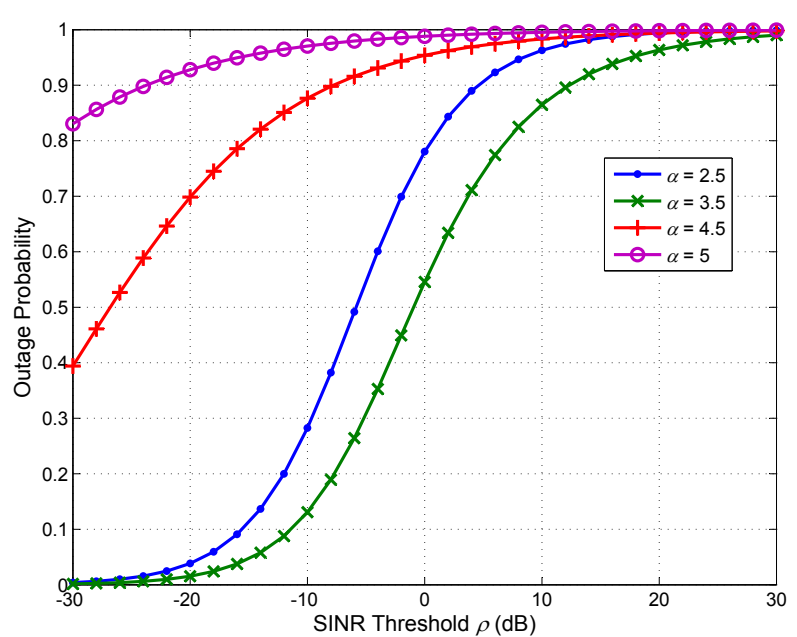

Fig. 3. Outage probability as a function of SINR threshold $\rho$.

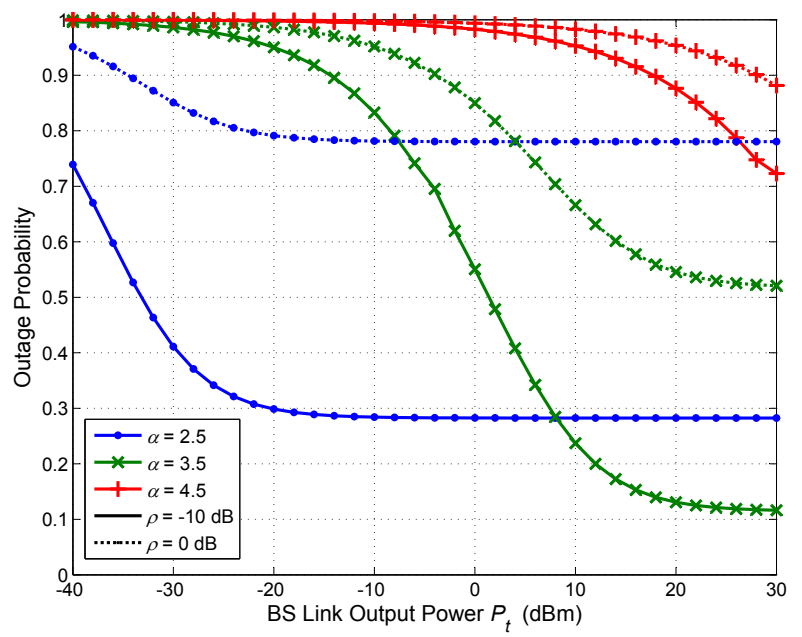

Fig. 4. Outage probability as a function of BS link output power $P_{t}$.

The next numerical results concern the scenario of the modified path loss model PL 2 with BS antenna height $L=30 \mathrm{~m}$. The main objective here is to assess the impact of the existing singularity that the commonly adopted $P L 1$ presents for small distances. In this context, the absolute error of outage probability by comparing the two models is illustrated in Fig.5 as a function of BS intensity $\lambda_{b}$, for different values of $a$ and $\rho$. All the curves have a similar behavior, where the error increases almost linearly with respect to $\lambda_{b}$, i.e. as the network becomes more dense. As this figure shows, the deviation between the two models is larger for higher values of $\rho$ and $a$, where the optimistic results are always provided by the $P L 1$. However, one can observe that this error is relatively small in all cases for the assumed set of parameters. For instance, the deviation is below $[2.5,1.1,0.3] \%$ for $\mathrm{BS}$ intensity $\lambda_{b}=[10,4,1]$ points $/ \mathrm{km}^{2}$ respectively. This marginal difference verifies the validity of Corollary 1 and admits the evaluation of EE gains based on the property presented therein. At this point, one should keep in mind that the Corollary 1 just provides a way to easily determine, in the case of sleep 


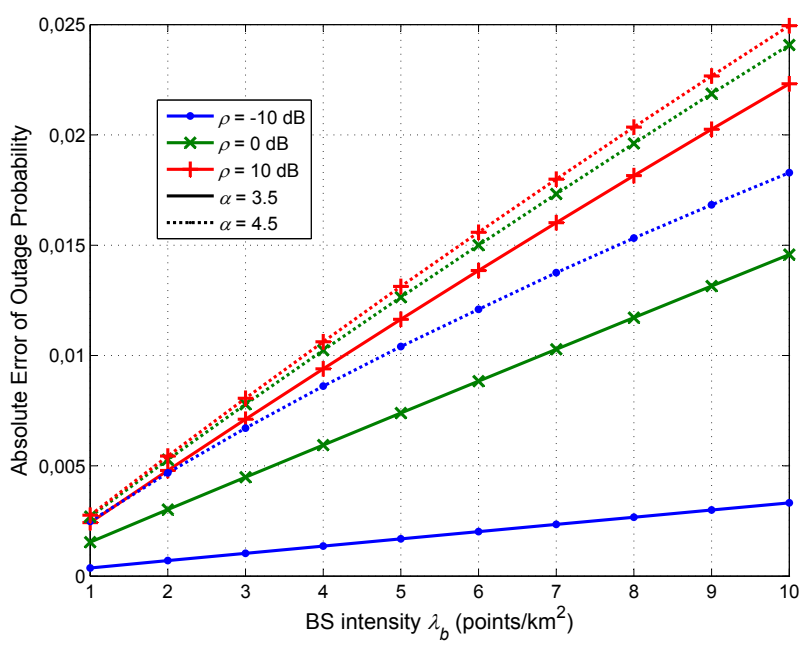

Fig. 5. Absolute error of outage probability due to singularity of path loss model as a function of BS intensity $\lambda_{b}$.

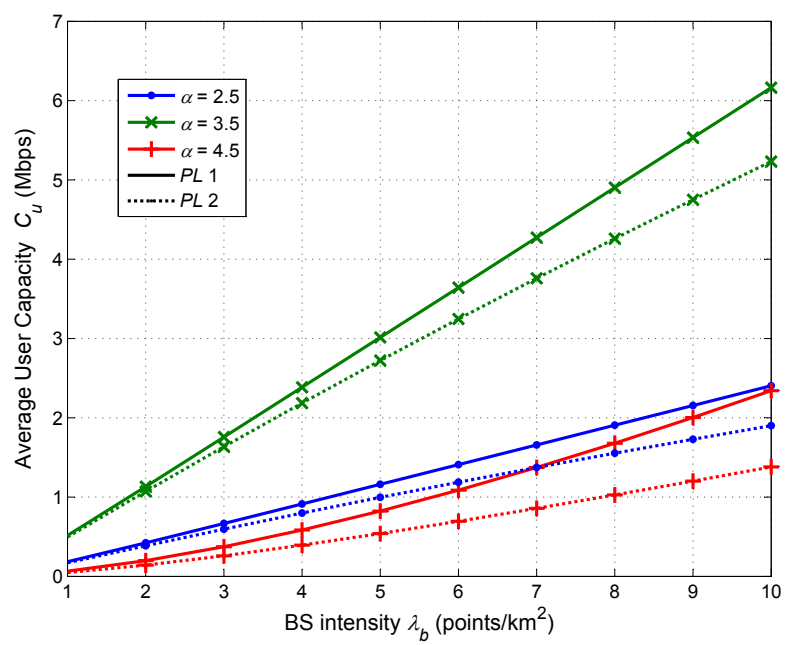

Fig. 6. Average user capacity $C_{u}$ as a function of BS intensity $\lambda_{b}$ for both singular ( $P L 1)$ and non-singular ( $P L 2)$ path loss model.

mode, the value of $P_{t}$ that achieves the same outage probability. Nevertheless, this can also be done under any scenario by the numerical computation of the corresponding integral, as for example in (18) for $P L 2$, and then the remainder of the analysis is exactly the same.

In the same framework, Fig. 6 demonstrates the respective average user capacity $C_{u}$ as a function of $\lambda_{b}$ for both path loss models according to (15),(19). The achieved capacity grows practically linearly with the number of deployed BSs and for reasons already explained, the curves for the intermediate value of $a=3.5$ present the best performance. Similarly to the case of the outage probability, the difference between the models can be neglected for low values of $\lambda_{b}$ in compliance with Corollary 2. As expected, higher capacity is achieved for $P L 1$, e.g. for $a=3.5, C_{u}=[0.52,2.38,6.16] \mathrm{Mbps}$ for $\lambda_{b}=[1,4,10]$ points $/ \mathrm{km}^{2}$ respectively.

Now that the basic issues for outage are examined, our interest turns to the energy savings from sleep mode and

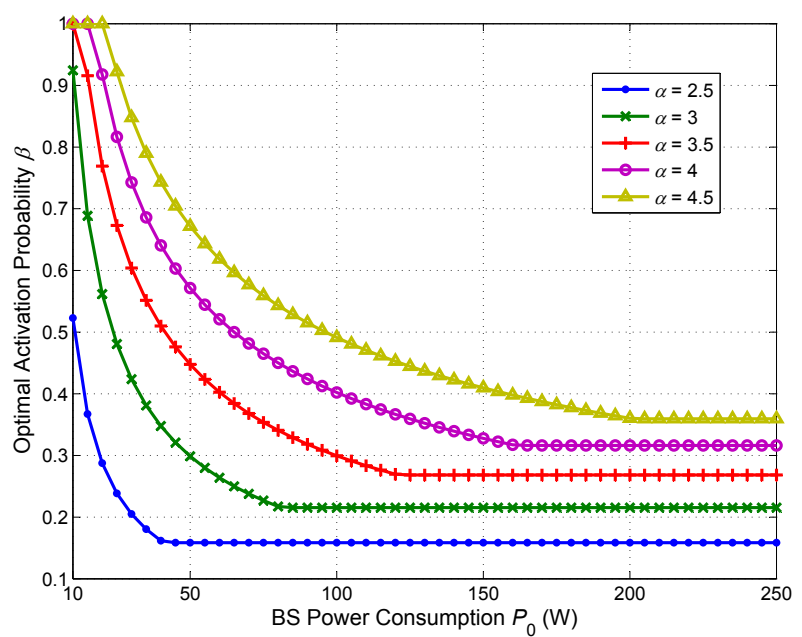

Fig. 7. Optimal activation probabilities $\beta_{o p t}$ as a function of BS power consumption $P_{0}$ and exponent $a$.

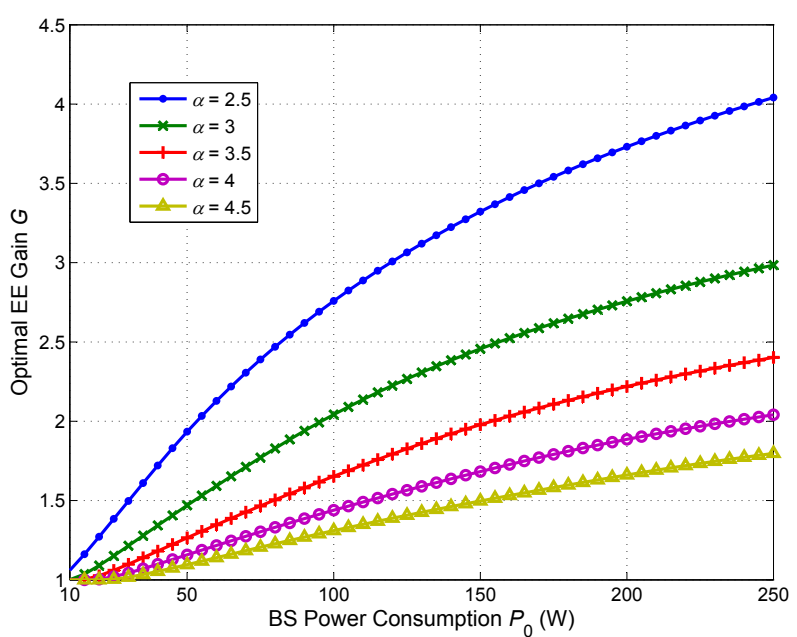

Fig. 8. Optimal EE gains $G$ as a function of BS power consumption $P_{0}$ and exponent $a$.

the respective optimal activation probabilities, focusing on the impact of the power model parameters $P_{0}, \gamma$ and $P_{\text {out }}$. Notice that $P_{\text {sleep }}$ can easily be examined as well, but it is omitted since $E E_{\text {sleep }}$ actually depends on the expression $P_{0}-P_{\text {sleep }}$.

Fig.7 presents the optimal activation probabilities $\beta_{\text {opt }}$ for different values of $P_{0}$, i.e. the minimum power consumption in active mode. All curves show similar behavior indicating that as $P_{0}$ increases, the optimal probability $\beta_{\text {opt }}$ progressively decreases. This is quite expected, since for higher values of $P_{0}$, the energy savings from sleep mode outperform the corresponding losses from the increased transmit power of the remaining BSs, and hence favor the selection of less active BSs. These gains are lower for worse propagation conditions, i.e. higher value of $a$. This behavior holds until a break point (horizontal part of curves), where the constraint for maximum transmit power takes place and $\beta_{\text {opt }}$ cannot be further reduced. The respective EE gains are shown in Fig.8 where it is clear that significant energy gains can be achieved for all cases. 


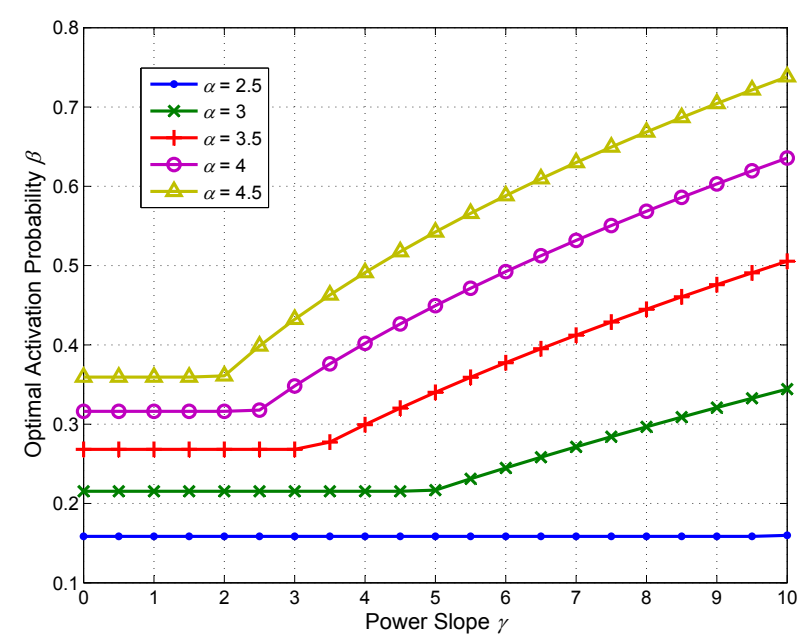

Fig. 9. Optimal probabilities $\beta_{\text {opt }}$ as a function of power slope $\gamma$.

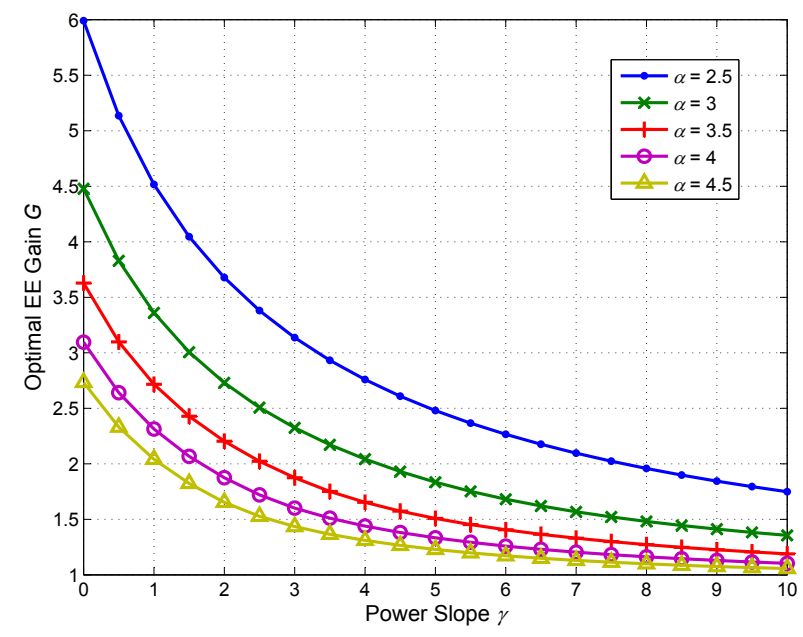

Fig. 10. Optimal EE gains $G$ as a function of power slope $\gamma$.

For instance, for $a=2.5$ and $P_{0}$ ranging from $P_{0}=50 \mathrm{~W}$ to $P_{0}=250 \mathrm{~W}$, the gains are increased from $G=1.93$ to $G=4.04$ with $16 \%$ active BSs. These savings are substantially reduced for the worst case of $a=4.5$, i.e. from $G=1.10$ with $\beta_{o p t}=0.67$ to $G=1.80$ with $\beta_{o p t}=0.36$ and for all the other values of $a$, the results lie between the previous two curves. The opposite behavior is noticed when the power slope $\gamma$ becomes the parameter under study. According to Figs.9-10, EE is reduced as $\gamma$ admits higher values. This is explained by the fact that the cost for the increased transmit power now becomes the dominant factor. For $a=2.5$ and power slope ranging from $\gamma=0$ to $\gamma=10$, the gains are decreased from almost $G=6$ to $G=1.75$ with $\beta_{\text {opt }}=0.16$. The respective gains for the worst conditions of $a=4.5$ range from $G=2.73$ with $\beta_{o p t}=0.36$ to nearly $G=1.06$ with $\beta_{o p t}=0.74$, where the latter one indicates that there is almost no gain provided.

The optimal activation probabilities $\beta_{o p t}$ and the respective energy gains for variable transmit power $P_{\text {out }}$ are depicted in Figs.11-12. The main difference here, as shown in Fig.11, is that the constraint for the maximum transmit power does

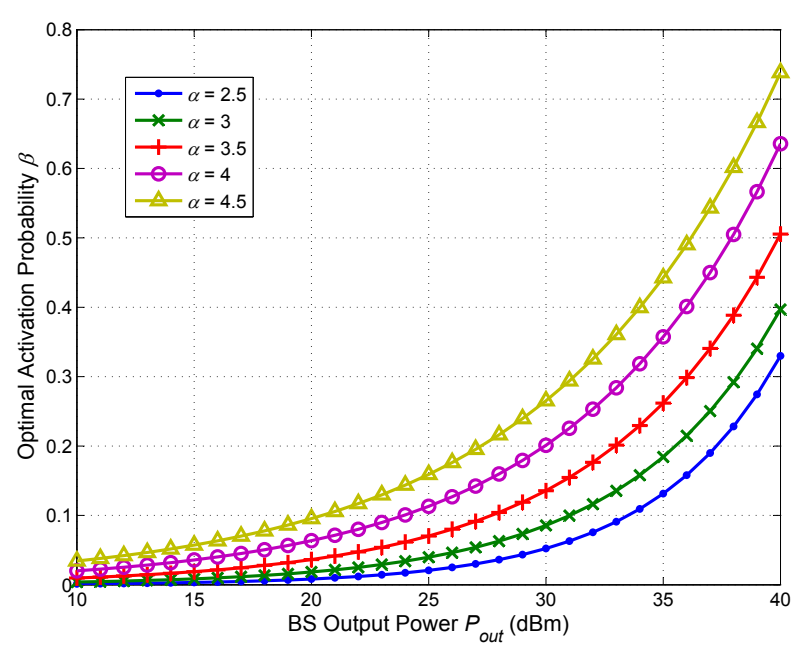

Fig. 11. Optimal probabilities $\beta_{\text {opt }}$ as a function of output power $P_{\text {out }}$.

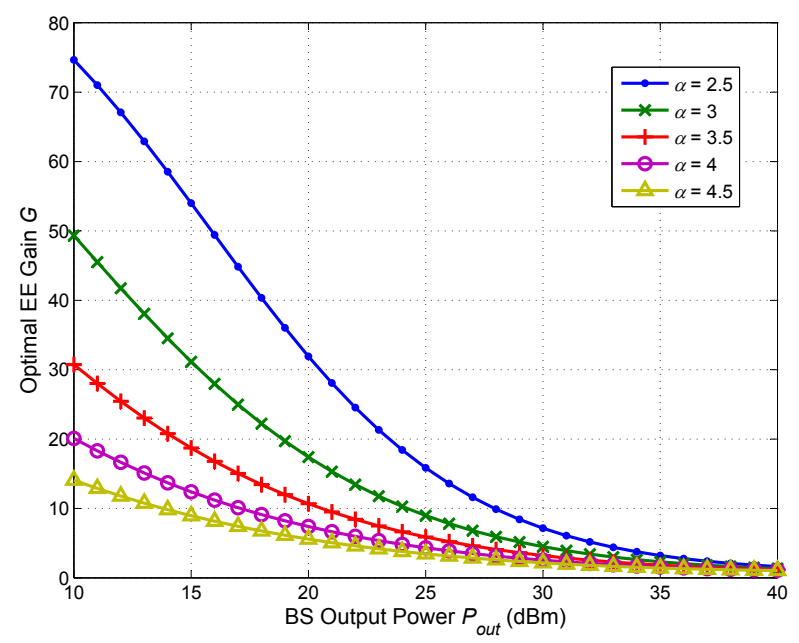

Fig. 12. Optimal EE gains $G$ as a function of output power $P_{\text {out }}$.

not depend on the value of $P_{\text {out }}$. This can also be explained from (23), where $P_{\text {out }}$ is a common factor of the arguments inside the max function, and thus does not affect its outcome. More importantly, as expected and illustrated in Fig.12, $P_{\text {out }}$ has a strong impact on the optimal gains. For example, if $P_{\text {out }}=35 \mathrm{dBm}$, then the EE gains lie between $G=3.21$ and $G=1.41$ with respect to $a$, while the respective savings for lower output power $P_{\text {out }}=30 \mathrm{dBm}$ range from $G=7.16$ to $G=2.15$. These gains grow dramatically as $P_{\text {out }}$ decreases, verifying that in low loading scenarios, the concept of BS sleep mode comprises a key feature in order to dramatically reduce network energy consumption.

Finally, the impact of sleep mode on the average user capacity $C_{u}$ is studied in the last part of the results. As explained previously, since our approach targets on achieving a specific level of outage probability, $C_{u}$ is reduced as fewer BSs remain active. In order to visualize this dependency, Fig.13 shows $C_{u}$ from (15) as a function of exponent $a$ for three different cases of probability $\beta$. Keep in mind that these results are carried out with $P_{t}$ increased by $\beta^{1-\frac{a}{2}}$ according 


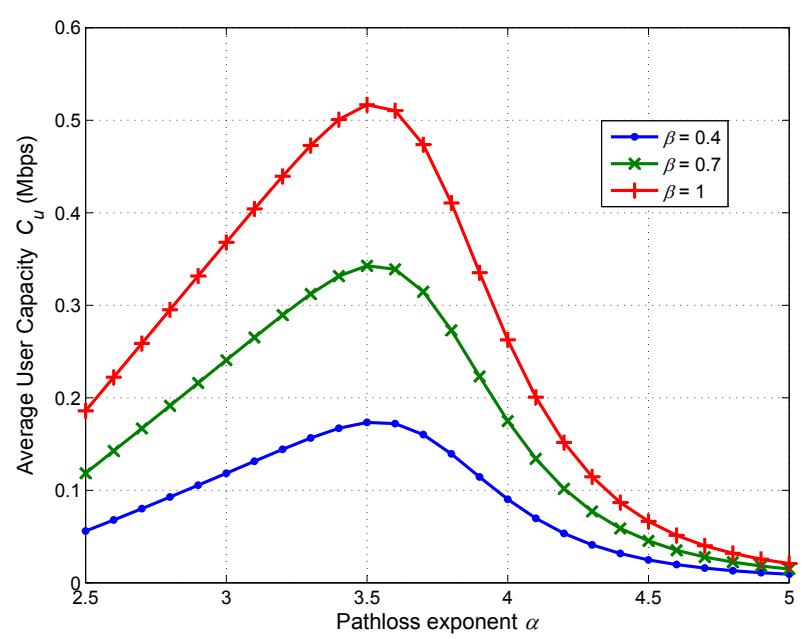

Fig. 13. Average user capacity $C_{u}$ as a function of path loss exponent for different values of activation probability $\beta$.

to Corollary 1. Similar behavior is noticed for all the curves where the maximum rate is achieved for $a_{\text {opt }}=3.5$. The exact value of $a_{\text {opt }}$ depends on the parameters from Table I, but in any case, $C_{u}$ is not a monotonic function of $a$ due to the opposing SNR and SIR terms and thus, an optimal value $a_{\text {opt }}$ always exists. As this figure illustrates, $C_{u}$ is reduced for lower values of $\beta$, especially for $a$ near $a_{\text {opt }}$ where in particular, for $a=3.5$, $C_{u}=[517,343,173] \mathrm{Kbps}$ for $\beta=[1,0.7,0.4]$ respectively. In general, if $C_{u}$ must be higher than a specified requirement, then a minimum allowed value of $\beta$ can easily be defined.

\section{CONCLUSIONS}

Motivated by the emerging need for green radio networking, the authors study in this work the optimal energy savings in an OFMDA cellular network with sleep mode, by exploiting useful properties of stochastic geometry. In this line of thought, the contribution of this paper is summarized by the following points: Firstly, a theoretical model that quantifies the key metrics of the outage probability and the average user capacity is extended in order to introduce the effect of sleep mode. Then, the case of a non-singular path loss function is examined in order to verify the validity of the analysis, since the most commonly used model can lead to inaccurate results. Furthermore, a sound framework that allows the evaluation of the optimal energy savings by reducing the number of active BSs in a way that the outage probability remains the same is proposed. In this context, the impact of this approach on the achieved user capacity is also investigated. Finally, an attempt to analyze the performance of the presented methodology for key parameters of the BS power consumption model is carried out through extended numerical computations.

These initial results encourage our study for further research, towards more sophisticated power, traffic and deployment models, that includes for example scenarios of heterogeneous networks and cooperating providers, and moreover focuses in detail on the existing spectral and EE trade-off, accounting for the challenging issue of planning green cellular networks.

\section{ACKNOWLEDGMENTS}

The work in this paper has been developed in the context of the GreenTouch initiative [4], (http://www.greentouch.org).

\section{REFERENCES}

[1] ITU-T, Environment \& Climate Change, "ITU News: Tackling Climate Change," ITU-T Climate Change Reports, Nov. 2011.

[2] NGMN Green Telco Initiative - Energy Efficiency and Green Footprint Activities, http://www.ngmn.org/workprogramme/greeninitiave.

[3] EARTH, "Energy aware radio and network technologies," European Research Project, http://www.ict-earth.eu.

[4] GreenTouch Initiative, http://www.greentouch.org.

[5] S. Mclaughlin et al., "Techniques for improving cellular radio base station energy efficiency," IEEE Wireless Commun. Mag., vol. 18, no. 5, pp. 1017, Oct. 2011.

[6] R. Wang, J. S. Thompson, H. Haas, and P. M. Grant, "Sleep mode design for green base stations," IET Commun., vol. 5, no. 18, pp. 2606-2616, Dec. 2011.

[7] I. Ashraf, F. Boccardi, and L. Ho, "SLEEP mode techniques for small cell deployments," IEEE Commun. Mag., vol. 49, no. 8, pp. 72-79, Aug. 2011.

[8] M. A. Marsan, L. Chiaraviglio, D. Ciullo, and M. Meo, "Switch-Off Transients in Cellular Access Networks with Sleep Modes," in Proc. of IEEE ICC'11, Kyoto, Japan, Jun. 2011.

[9] E. Altman, M. K. Hanawal, R. El-Azouzi, and S. Shamai, "Tradeoffs in green cellular networks," SIGMETRICS Perform. Eval. Rev., vol. 39, no. 3, pp. 67-71, Dec. 2011

[10] L. Saker, S. E. Elayoubi, and H. O. Scheck, "System Selection and Sleep Mode for Energy Saving in Cooperative 2G/3G Networks," in Proc. of IEEE VTC'09-Fall, Anchorage, USA, Sep. 2009.

[11] M. A. Marsan and M. Meo, "Energy Efficient Management of two Cellular Access Networks," SIGMETRICS Perform. Eval. Rev., vol. 37, no. 4, pp. 69-73, Mar. 2010.

[12] C. Bae and W. Stark, "End-to-End Energy-Bandwidth Tradeoff in Multihop Wireless Networks," IEEE Trans. Inf. Theory, vol. 55, no. 9, pp. 4051-4066, Sep. 2009.

[13] D. Grace, J. Chen, T. Jiang, and P. D. Mitchell, "Using Cognitive Radio to Deliver 'Green' Communications," in Proc. of CROWNCOM'09, Hannover, Germany, Jun. 2009.

[14] T. Wang and L. Vandendorpe, "Sum Rate Maximized Resource Allocation in Multiple DF Relays Aided OFDM Transmission," IEEE J. Sel. Areas Commun., vol. 29, no. 8, pp. 1559-1571, Sep. 2011.

[15] D. Stoyan, W. S. Kendall, and J. Mecke, Stochastic Geometry and Its Applications, 2nd ed. John Wiley \& Sons, 1996.

[16] M. Haenggi et al., "Stochastic geometry and random graphs for the analysis and design of wireless networks," IEEE J. Sel. Areas Commun., vol. 27, no. 7, pp. 1029-1046, Sep. 2009.

[17] F. Baccelli and B. Baszczyszyn, "Stochastic Geometry and Wireless Networks Volume 1: THEORY," Foundations and Trends in Networking, vol. 3, no. 3-4, pp. 249-449, Dec. 2009.

[18] J. G. Andrews, F. Baccelli, and R. K. Ganti, "A Tractable Approach to Coverage and Rate in Cellular Networks," IEEE Trans. Commun., vol. 59, no. 11 , pp. 3122-3134, Nov. 2011.

[19] E. Cardis et al., "The INTERPHONE study: design, epidemiological methods, and description of the study population," European Journal of Epidemiology, vol. 22, no. 9, pp. 647-664, Jul. 2007.

[20] G. Auer et al., "Cellular Energy Efficiency Evaluation Framework," in Proc. of IEEE VTC'11-Spring, Budapest, Hungary, May 2011.

[21] O. Arnold, F. Richter, G. Fettweis, and O. Blume, "Power consumption modeling of different base station types in heterogeneous cellular networks," in Proc. of Future Network and Mobile Summit, 2010, Florence, Italy, Jun. 2010.

[22] Y. Chen, S. Zhang, and S. Xu, "Impact of Non-Ideal Efficiency on Bits Per Joule Performance of Base Station Transmissions," in Proc. of IEEE VTC'11-Spring, Budapest, Hungary, May 2011.

[23] E. Altman, C. Hasan, J.-M. Gorce, and L. Roullet, "Green networking: Downlink considerations," in Proc. of NetGCooP'11, Paris, France, Oct. 2011.

[24] H. Inaltekin, S. B. Wicker, M. Chiang, and H. V. Poor, "On Unbounded Path-Loss Models: Effects of Singularity on Wireless Network Performance," IEEE J. Sel. Areas Commun., vol. 27, no. 7, pp. 1078-1092, Sep. 2009. 